\title{
Caracterização térmica do poliuretano derivado de óleo vegetal utilizado para a confecção de dispositivo de assistência ventricular
}

\author{
J. E. Carvalho ${ }^{1 *}$, G. O. Chierice ${ }^{2}$; S. Claro Neto ${ }^{2}$ \\ ${ }^{I}$ Departamento de Pós-Graduação em Ciência e Eng. de Materiais - USP, São Carlos - SP, Brasil \\ ${ }^{2}$ Instituto de Química de São Carlos, Universidade de São Paulo, Av. Trabalhador são-carlense, 400 - 13560-970 São Carlos/SP, Brazil
}

Received 08/05/2014; accepted 02/06/2014

Available online 27/08/2014

\begin{abstract}
This study is the characterization of a polyurethane derived from vegetable oil for the by extracorporeal artificial heart, using techniques as thermogravimetric analysis (TGA) and dynamic mechanical analysis (DMA). The proposed objective was to obtain information of the polymer, proving its efficiency as a biomaterial that will be submitted to the working conditions of an extracorporeal heart; thermal stability of the body human temperature $\left(36-40^{\circ} \mathrm{C}\right)$ and mechanical, simulating the functions of a mechanical artificial heart device with extracorporeal oxygenation.
\end{abstract}

Keywords: Polyurethane, vegetable oil, artificial heart

\section{Introdução}

O poliuretano derivado do óleo de vegetal é considerado um material atóxico, por não liberar substâncias prejudiciais aos organismos vivos. Este poliuretano faz parte de uma classe de polímeros à base de moléculas de óleo de mamona, desenvolvido em 1984 por pesquisadores do grupo de Química Analítica e Tecnologia de Polímeros de São Carlos (GQTP) - Universidade de São Paulo [1].

Os polímeros utilizados na área médica vêm substituindo, com sucesso, outros materiais no desenvolvimento de implantes e dispositivos para a utilização temporária ou definitiva [2]. O poliuretano derivado de óleo vegetal é um biopolímero que vem ao encontro dessa tendência, utilizado na área médica devido às suas propriedades termodinâmica e biológica serem compatíveis com o mecanismo de funcionamento do corpo humano.

O poliuretano estudado neste trabalho é derivado de um poliol com segmentos do poliéster sintetizado a partir de óleo vegetal (óleo de mamona) e um pré-polímero sintetizado a partir dos poliois com difenilmetanodiisocianato. Os óleos vegetais, que são triglicerídeos de ácidos graxos, hidroxilados ou não fornecem excelente plataforma para síntese de materiais poliméricos. Além disso, apresentam baixa toxicidade, menores quantidades de formação de resíduos durante a fase de processamento e os produtos obtidos são, geralmente biodegradáveis, por consequência, menos poluentes. Esse polímero vegetal é considerado como um material termofixo, que possui ligações cruzadas covalentemente entre as cadeias moleculares adjacentes. Essas características constituem um forte atrativo para a sua utilização como matéria-prima para a indústria química [므르.
O grau de polimerização do poliuretano pode ser considerado completo, quando suas propriedades térmicas e viscoelásticas, que podem ser estudadas utilizando as técnicas termogravimétricas (TGA) e análise dinâmicomecânica (DMA), adquire valores constantes, não sofrendo mais mudanças significativas com o tempo. No caso da TGA, quando o polímero completa a tempo de polimerização, é observado que o seu processo de degradação térmica adquire um comportamento onde a curva termogravimétrica se apresenta com etapas de perdas de massa bem definidas, que passa a ser uma característica deste material [6]. O mesmo acontece com as propriedades viscoelásticas obtidas nas curvas DMA. Estudando essas propriedades, é possível avaliar aplicabilidade do poliuretano como biomaterial para confecção do coração extracorpóreo.

\subsection{Análise Térmica}

A análise térmica engloba um conjunto de técnicas muito utilizadas para caracterização de polímeros, pois podem determinar propriedades que estão diretamente relacionadas à sua estrutura molecular. $\mathrm{O}$ uso dessas técnicas é importante, principalmente para caracterizar propriedades que podem comprometer o desempenho de um material durante a sua aplicação. Como no caso em que um polímero é submetido a esforços mecânicos e temperatura, de maneira a poder ocasionar a liberação de substâncias por processos de degradação térmica ou ainda mudar suas propriedades viscoelásticas, inviabilizando sua utilização. Trabalhos realizados por Claro Neto []], Cangemi [ㅁ, $\underline{9}$ ], Trovati [10], tem demonstrado que os poliuretanos derivados de óleo vegetal possuem uma estabilidade térmica cuja degradação inicia acima de $200{ }^{\circ} \mathrm{C}$ e as suas propriedades viscoelásticas estão relacionadas a formulação estabelecida durante a síntese do poliol e do pré-polímero. 
Neste trabalho, serão realizados estudos analíticos com amostras de poliuretano derivado de óleo vegetal utilizando a técnica TGA e DMA. A análise termogravimétrica (TGA) é uma técnica que fornece informações quantitativas sobre a estabilidade térmica do material, proporcionando uma avaliação de seu processo de degradação térmica [7]. Com a utilização da técnica DMA, é possível determinar os módulos mecânicos de viscoelasticidade do material, que é um comportamento dependente de sua estrutura molecular, o que permite avaliar o grau de polimerização das amostras e sua temperatura de transição vítrea. Por meio desses resultados, é possível prever o desempenho do poliuretano quando utilizado em dispositivos médicos, podendo evitar, assim, qualquer tipo de deformação termomecânica.

\section{Experimental}

\subsection{Termogravimetria (TGA)}

A Termogravimetria (TGA) foi efetuada no GQATP/IQ - USP São Carlos, com amostras de poliuretano derivado de óleo vegetal, confeccionadas a partir de um prépolímero e um poliol na relação estequiométrica 1:1, em massa. Essas amostras foram submetidas em três condições distintas de polimerização; (amostra 1 - PU) tempo de polimerização de 6 horas (cura incompleta) em temperatura ambiente, (amostra 2 - PU tratada) após ter sido submetida a tratamento térmico por 24 horas, a $90{ }^{\circ} \mathrm{C}$ e (amostra 3 - PU envelhecida) após 1 ano de envelhecimento a temperatura ambiente. As curvas termogravimétricas foram obtidas na termobalança modelo Q50 da TA Instruments, utilizando-se cerca de 6,0 mg de amostra em porta amostra de platina. A razão de aquecimento foi de $10{ }^{\circ} \mathrm{C} \min ^{-1}$ na faixa de temperatura de 25 a $1000{ }^{\circ} \mathrm{C}$, em atmosfera de $\mathrm{N}_{2}$ com fluxo de $100 \mathrm{~mL} \mathrm{~min}^{-1}$.

\subsection{Análise dinâmico-mecânica (DMA)}

Para os ensaios DMA foram utilizados corpos de prova retangulares de poliuretano, confeccionados a partir de um pré-polímero e um poliol na relação estequiométrica 1:1, em massa. O primeiro, após ter sido retirado do molde, para completar a polimerização, ficou em repouso na temperatura ambiente durante um período de três dias e o segundo, após ter sido retirado do molde, para completar a polimerização, foi feito um tratamento térmico, onde ficou em uma estufa a $90{ }^{\circ} \mathrm{C}$ por 24 horas.

Após o preparo dos corpos de prova, esses foram submetidos ao ensaio de DMA, realizados no GQATP/IQ - USP São Carlos. Estes ensaios foram realizados no equipamento DMA Q800 da TA Instruments, com sistema de garra Dual Cantilever, seguindo a norma ASTMD5418-07 [11]. As condições foram realizadas com frequência de oscilação de $1 \mathrm{~Hz}, 0,25 \%$ de deformação, amplitude $20 \mu \mathrm{m}$ e razão de aquecimento de $3^{\circ} \mathrm{C} \min ^{-1}$ na faixa de aquecimento de 25 a $150{ }^{\circ} \mathrm{C}$. Os corpos de prova foram confeccionados com as dimensões aproximadas de $60,0 \times 13,80 \times 3,80 \mathrm{~mm}$.

\section{Resultados e Discussão}

\subsection{Termogravimetria (TGA)}

A Figura 1 mostra as curvas obtidas das amostras que foram confeccionadas em tempos de polimerização e temperaturas distintos e sumarizam os três experimentos termogravimétricos.

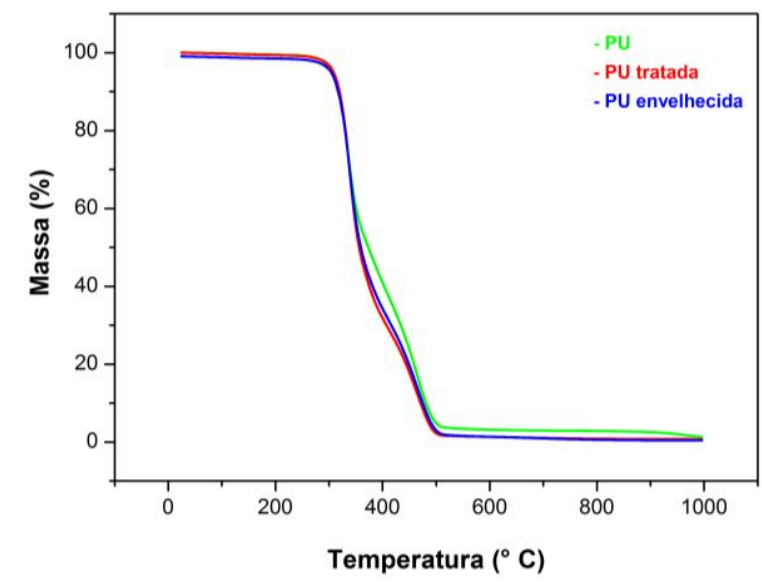

Figura 1: Sumarização das curvas TGs das amostras com distintos tempos de polimerização.

$\mathrm{Na}$ Figura 1, observam-se duas etapas de decomposição térmica nas três amostras realizadas. As análises de todas elas ocorreram na mesma faixa de temperatura, que corresponde, a primeira etapa de decomposição térmica entre 229 a $410{ }^{\circ} \mathrm{C}$. Essa decomposição está relacionada com a quebra da ligação uretano e perda do difenilmetano presente no pré-polímero [7]. A segunda etapa ocorre numa faixa de temperatura entre 410 a $558{ }^{\circ} \mathrm{C}$, estando relacionado à quebra dos ésteres graxos presente no poliol. As curvas termogravimétricas da amostra PU tratada (linha vermelha) diferencia-se das demais por apresentar a primeira etapa de decomposição uma perda de massa de $70 \%$, e a segunda etapa uma perda de massa de $28 \%$.

Na tabela 1 são mostrados os resultados das análises da primeira etapa representada por perda de massa 1 $\left(\Delta \mathrm{m}_{1}\right)$ e a segunda etapa pela perda de massa $2\left(\Delta \mathrm{m}_{2}\right)$.

Tabela 1: Resultados obtidos a partir da técnica termogravimétrica.

\begin{tabular}{ccc}
\hline \multirow{2}{*}{ AMOSTRAS } & $\begin{array}{c}\Delta \mathrm{m}_{\mathbf{1}} \\
(\boldsymbol{\%})\end{array}$ & $\begin{array}{c}\Delta \mathrm{m}_{\mathbf{2}} \\
(\boldsymbol{\%})\end{array}$ \\
\hline PU & 62 & 34 \\
PU TRATADA & 70 & 28 \\
PU ENVELHECIDA & 67 & 30 \\
\hline
\end{tabular}

A partir desses resultados, observou-se que aumento da porcentagem na primeira etapa, de perda de massa nas amostras que passou por tratamento térmico e a que foi envelhecida pelo tempo está coerente com o processo de polimerização do poliuretano, visto que nos dois casos houve um aumento da quantidade de ligações uretanos. Em contra partida, houve uma diminuição na porcentagem de perda na segunda etapa, como seria previsto 
em um processo de polimerização. Nas três amostras avaliadas não se observou resíduo final.

\subsection{Análise dinâmico-mecânica (DMA)}

As curvas DMA são resultados do comportamento viscoelástico do poliuretano em função da temperatura. Como o comportamento viscoelástico se apresenta na forma de duas componentes (componentes elástica - módulo de elasticidade de armazenamento E` e componente viscosa - módulo de elasticidade de perda E“), as Figuras 2 e 3 mostram as curvas com essas duas componentes e mais uma terceira curva que é a razão entre E“/E', também em função da temperatura, conhecida como curva de amortecimento ou Tan delta $(\delta)$.

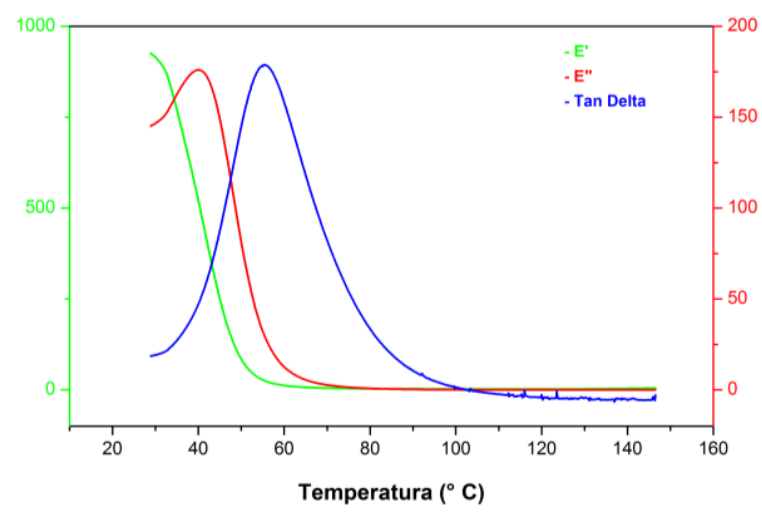

Figura 2: Curva DMA para amostra sem tratamento térmico.

Como o poliuretano está sendo desenvolvido para trabalhar a $36{ }^{\circ} \mathrm{C}$, nesta temperatura, para amostra que não passaram por tratamento térmico, é observado na Figura 2 o valor para módulo de elasticidade de armazenamento (E') de 892 Mpa e para o módulo de elasticidade de perda (E”) de 204,1 Mpa, enquanto que o pico da curva Tan delta $(\delta)$, que determina a temperatura de transição vítrea do material, o valor de $56,13{ }^{\circ} \mathrm{C}$.

Na Figura 3 são mostradas as curvas para o módulo de armazenamento E’, módulo de perda E“ e Tan delta $(\delta)$ em função da temperatura para amostra de poliuretano que passou por tratamento térmico.

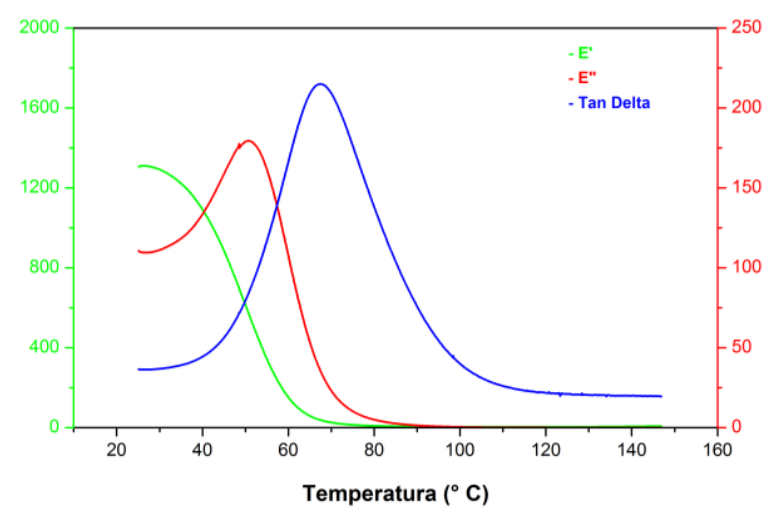

Figura 3: Curva DMA para amostra com tratamento térmico.

De acordo com a Figura 3, a $36{ }^{\circ} \mathrm{C}$, o valor encontrado para o módulo de elasticidade de armazenamento
(E') é de $1201 \mathrm{MPa}$, e para o módulo de elasticidade de perda (E") é de 98,08 $\mathrm{MPa}$, enquanto que para o pico da curva Tan delta $(\delta)$ é de $68,30^{\circ} \mathrm{C}$.

Comparando as curvas das Figuras 2 e 3, observa-se que houve um aumento na rigidez do poliuretano devido ao aumento do valor do módulo de elasticidade de armazenamento, acompanhado de um aumento na temperatura de transição vítrea. Essa mudança é característica de um aumento do grau de polimerização, que está relacionado a um aumento do entrecruzamento da estrutura molecular.

\section{Conclusão}

Os resultados deste trabalho demonstram que o poliuretano derivado de óleo vegetal é estável termicamente na temperatura de 36 a $40 \mathrm{oC}$, comprovando pela técnica termogravimétrica (TGA) utilizada quantitativamente a não liberação de qualquer substância que prejudique o organismo vivo durante a utilização do poliuretano derivado de óleo vegetal. Pela técnica de DMA, os resultados mostraram que o tratamento térmico auxilia na polimerização, fornecendo mais energia aos movimentos intramoleculares do biopolímero, facilitando o choque efetivo entre os sítios ativos. Esses choques aumentam o grau de polimerização do material, que, além de não deixar funções livres que possam interagir quimicamente com o organismo, aumentam também sua temperatura de transição vítrea.

\section{Referencias}

[1] Chierice GO. Pesquisa e desenvolvimento de biomateriais, baseados em poliuretanas derivadas de óleo de mamona. SNM - PADCT v.II 1994;76. [Google Scholar]

[2] Carvalho JE, Claro Neto S, Chierice GO. Avaliação da degradação do poliuretano derivado de óleo vegetal para confecção de coração artificial extracorpóreo. In: VI Encontro dos usuários das Técnicas Termoanalíticas. São Carlos. IQSC/USP; 2013. [Google Scholar]

[3] Saran WR. Estudo experimental de implantes derivados da resina poliuretana de mamona (ricinus communis), inseridos no canal medular da tíbia de coelhos. Análise da interface osso e implante. Dissertação (Mestrado) - Escola de Engenharia de São Carlos, Universidade de São Paulo, São Carlos, 2006. [Visualizar Item]

[4] Pereira PHL. Estudo das propriedades físicoquímicas da poliuretana derivada do óleo mamona com potencial aplicação na área médica. Dissertação (Mestrado) - Instituto de Química de São Carlos, Universidade de São Carlos, São Carlos, 2010. [Visualizar Item]

[5] Canevarolo Jr SV. Ciências dos Polímeros: Um texto básico para tecnológicos e engenheiros. São Paulo. Artiliber; 2002. [Google Scholar] 
[6] Carvalho JE, Claro Neto S, Chierice GO. Influência do tratamento térmico nas propriedades termomecânicas do poliuretano derivado do óleo vegetal. In: $1^{\circ}$ Encontro de Ciência e Engenharia de Materiais de São Carlos: Energia, Sustentabilidade e Inovação. São Carlos, UFSCAR, 2013.

[7] Claro Neto S. Caracterizações físico-químicas de um poliuretano derivado de óleo de mamona utilizado para implantes ósseos. Tese (doutorado) Instituto de Química São Carlos, Universidade de São Paulo, São Carlos, 1997.

[8] Cangemi JM, Claro Neto S, Chierice GO, Santos AM. Study of the biodegradation of polymer derived from castor oil by electron microscopy, thermogravimetry and infrared spectroscopy. Polímeros - Ciência e Tecnologia, Brasil 2006:16(2):129-135. [Google Scholar] [Visualizar Item] [CrossRef]

[9] Cangemi JM, Santos AM, Claro Neto S. Biodegradação: uma alternativa para minimizar os impactos decorrentes dos resíduos plásticos. Quí. Nov. Esc. 2005:22:17-21. [Google Scholar]

[10] Trovati G, Sanches EA, Claro Neto S, Mascarenhas YP, Chierice GO. Characterization of polyurethane resins by FTIR, TGA, and XRD. J. Appl. Polym. Sci. 2010:115(1):263-268. [Google Scholar] [Visualizar Item] [CrossRef]

[11] American Society For Testing And Materials. Standard Test Method for Plastics: Dynamic Mechanical Properties: In Fluxure (Dual Cantilever Beam). ASTM D5418-07. [CrossRef] 